\title{
Prognostic Analysis and Comparison of the 2014 and 2018 International Federation of Gynecology and Obstetrics Staging System on Overall Survival in Patients with Stage IIB-IVA Cervix Carcinoma
}

\author{
Tao Song $\mathbb{D}^{1,2}$, Hong'en $\mathrm{Xu}^{2}$, Lei Shi ${ }^{2}$, Senxiang Yan' \\ 'Department of Radiation Oncology, The First Affiliated Hospital, Zhejiang University School of Medicine, Hangzhou, 3I0003, Zhejiang, People's \\ Republic of China; ${ }^{2}$ Cancer Center, Department of Radiation Oncology, Zhejiang Provincial People's Hospital, Affiliated People's Hospital, Hangzhou \\ Medical College, Hangzhou, Zhejiang, 3100I4, People's Republic of China \\ Correspondence: Senxiang Yan, Department of Radiation Oncology, The First Affiliated Hospital, Zhejiang University School of Medicine, No. 79, \\ Qingchun Road, Shangcheng District, Hangzhou, 310003, People's Republic of China, Tel +86 57I-87236403, Email yansenxiang@zju.edu.cn
}

\begin{abstract}
Purpose: This study aimed to assess the prognostic factors of overall survival (OS) in patients with stage IIB-IVA cervix carcinoma (CC) who underwent external beam radiation therapy (EBRT) and brachytherapy (BRT) and to compare the prognostic accuracy of the 2014 and 2018 International Federation of Gynecology and Obstetrics (FIGO) staging system using the Surveillance, Epidemiology, and End Results (SEER) database.

Methods: Patients with a histopathological diagnosis of CC between 2004 and 2016 were included. The primary endpoint was OS. The prognostic significance for OS was analyzed by the Cox regression model. Prognostic accuracy in evaluating 3- and 5-year OS in different staging systems was evaluated using time-dependent receiver operating characteristic (tdROC) curves.

Results: A total of 2585 patients with stage IIB-IVA CC, staged according to the 2014 FIGO staging system, were included in the study. The 3- and 5-year OS rates were $63.9 \%$ and 56.6\%, respectively, with a median OS of 98 months. Independent variables, such as older age at diagnosis, histological grades apart from well or moderately differentiated, large tumor size, advanced tumor stages classified according to the 2014 and 2018 FIGO staging systems and treatment without chemotherapy or unknown were associated with a worse OS. A tdROC analysis conducted using DeLong's tests revealed no significant difference in the prediction of 3- and 5-year OS between the 2014 and 2018 FIGO staging systems ( $P=0.912$ and 0.863 , respectively).

Conclusion: Both 2014 and 2018 FIGO staging systems were strong prognostic factors for OS. No significant risk classification was observed for stage IIIC1 disease in the revised 2018 FIGO staging system for patients who underwent EBRT and BRT.
\end{abstract}

Keywords: prognostic analysis, Surveillance, Epidemiology, and End Results database, staging, survival, risk factors

\section{Introduction}

Based on the updated statistics by the GLOBOCAN study, ${ }^{1}$ cervix carcinoma (CC) continues to rank as the fourth most common malignancy (6.5\%) in women. CC accounted for 341,831 cancer-related deaths in 2020, an increase from 266,000 deaths in 2012, worldwide. ${ }^{2}$ Among the stages of CC, the International Federation of Gynecology and Obstetrics (FIGO) stage IIB-IVA accounts for more than $80 \%$ of new diagnoses in developing countries. ${ }^{3,4}$ For these patients, external beam radiation therapy (EBRT) along with brachytherapy (BRT) and concomitant chemotherapy (CT) has been recommended as the paradigm of curative treatment based on a recent series of high level randomized controlled trials. ${ }^{5-7}$ However, $25-75 \%$ of patients with stage IIB-IVA disease may develop tumor recurrence or distant metastasis, ${ }^{8}$ which needs systemic therapy to palliate clinical symptoms and improve poor survival rates. ${ }^{9}$ Therefore, there is an urgent requirement to identify potential prognostic factors in patients with stage IIB-IVA CC to maximize the benefit of radiotherapy (RT) or concurrent chemo-radiotherapy (CCRT). 
Accurate tumor staging plays a vital role in deciding the upfront treatment of various cancers. ${ }^{10}$ It helps to decide different treatment intentions and potential treatment combinations per stage. For the staging of CC, FIGO updated its 2014 version in 2018. ${ }^{11,12}$ Compared with the 2014 FIGO staging system, the 2018 FIGO revision defined regional lymph node (LN) metastasis identified via pathological and/or radiological findings as a stage IIIC disease. ${ }^{13,14}$ The positive pelvic and para-aortic LN metastasis were specifically assigned to stage IIIC1 and stage IIIC2, respectively. Furthermore, this new staging system can classify patients based on different risk levels ${ }^{12}$ and has proved its better prediction accuracy in a cohort of 586 patients in China. ${ }^{15}$ However, some aspects, especially those for stage IIIC disease, remain controversial. ${ }^{16,17}$ Shin et al compared the survival outcomes of 2441 patients with $\mathrm{CC}$ based on the staging manual of the 2014 and 2018 FIGO staging systems from the Korean National Cancer Registry. ${ }^{18}$ In the subgroup analysis for stage IIIC $(\mathrm{n}=502)$, patients with stage IIIC1 disease were observed to have significantly better survival outcomes than those with stage IIIA/IIIB disease $(P<0.001)$.

Based on the above paradox, the aim of the current study was to explore the overall survival (OS) outcomes and potential prognostic factors of OS in patients with stage IIB-IVA CC based on the 2014 FIGO staging system who were registered to undergo EBRT and BRT and to further compare the utility of the 2014 and 2018 FIGO staging systems using data from the National Cancer Institute's Surveillance, Epidemiology, and End Results (SEER) database.

\section{Patients and Methods Study Population}

This study collected data from the 18 population-based registries of the SEER database (SEER * Stat 8.3.6). Data of 35,565 patients with CC from 2004 to 2016 were extracted from the database under the International Classification of Diseases for Oncology, 3rd Edition (ICD-O-3) recodes of C53.8-9. The major inclusion criteria included the following: 1) patients with a confirmed histopathological diagnosis of CC; 2) primary diagnosis of CC with IIB-IVA staging according to the 6th edition criteria of the TNM classification and the SEER database combined stage $(2016+)$ for patients diagnosed in 2016; 3) patients who underwent the combined treatment of EBRT and BRT. Patients with untraced or incomplete data and whose survival time was less than 1 month were excluded from the current study (Figure 1).

\section{Patient Variables}

Patient variables such as age at diagnosis, marital status, race, tumor histology, differentiation, tumor size, tumor stage, use of CT, survival data including survival status (surviving or non-surviving) and survival time in months were extracted from the database. Based on previous studies, 70 years at diagnosis was set as the cut-off point for age. ${ }^{19-21}$ Marital status was assorted as a binary factor by categorizing the patients as married (including common law), unmarried and others, which included marital status as divorced, separated, single, widowed, unmarried or domestic partner and unknown, as adopted in other SEER studies. ${ }^{22,23}$ Within the original SEER database, tumors were staged based on the American Joint Committee on Cancer (AJCC) TNM 6th edition for patients diagnosed between 2004 and 2015, ${ }^{24}$ and SEER database combined stage for patients registered in 2016. The algorithm for stages diagnosed after 2016 is available at: https://seer.cancer.gov/seerstat/variables/seer/ajcc-stage/seer-combined.html. Tumors were then restaged based on the stage definitions mentioned in the 2014 FIGO and 2018 FIGO editions. ${ }^{11,12}$ Notably, the para-aortic LN involvement was defined as distant metastasis (M1) in both the AJCC 6th edition and SEER database combined stage. Therefore, only the patients with stage IIIC1 disease with documented positive pelvic LNs were included in the present study.

\section{Outcome Analysis}

OS was calculated as the duration from the diagnosis of CC to death or the last follow-up registered in the database and was set as the primary endpoint of this study. Baseline characteristics of patients were summarized by descriptive statistics and frequency tables. The one-way analysis of variance (ANOVA) and Bonferroni correction were used to compare the proportions of different tumor groups. Survival curves were estimated using the Kaplan-Meier method along with the Log rank test. Univariate and multivariate analysis using the Cox regression model were performed as previously described. ${ }^{25}$ To further evaluate the prediction accuracy of the two different FIGO staging systems, the time-dependent receiver operating 


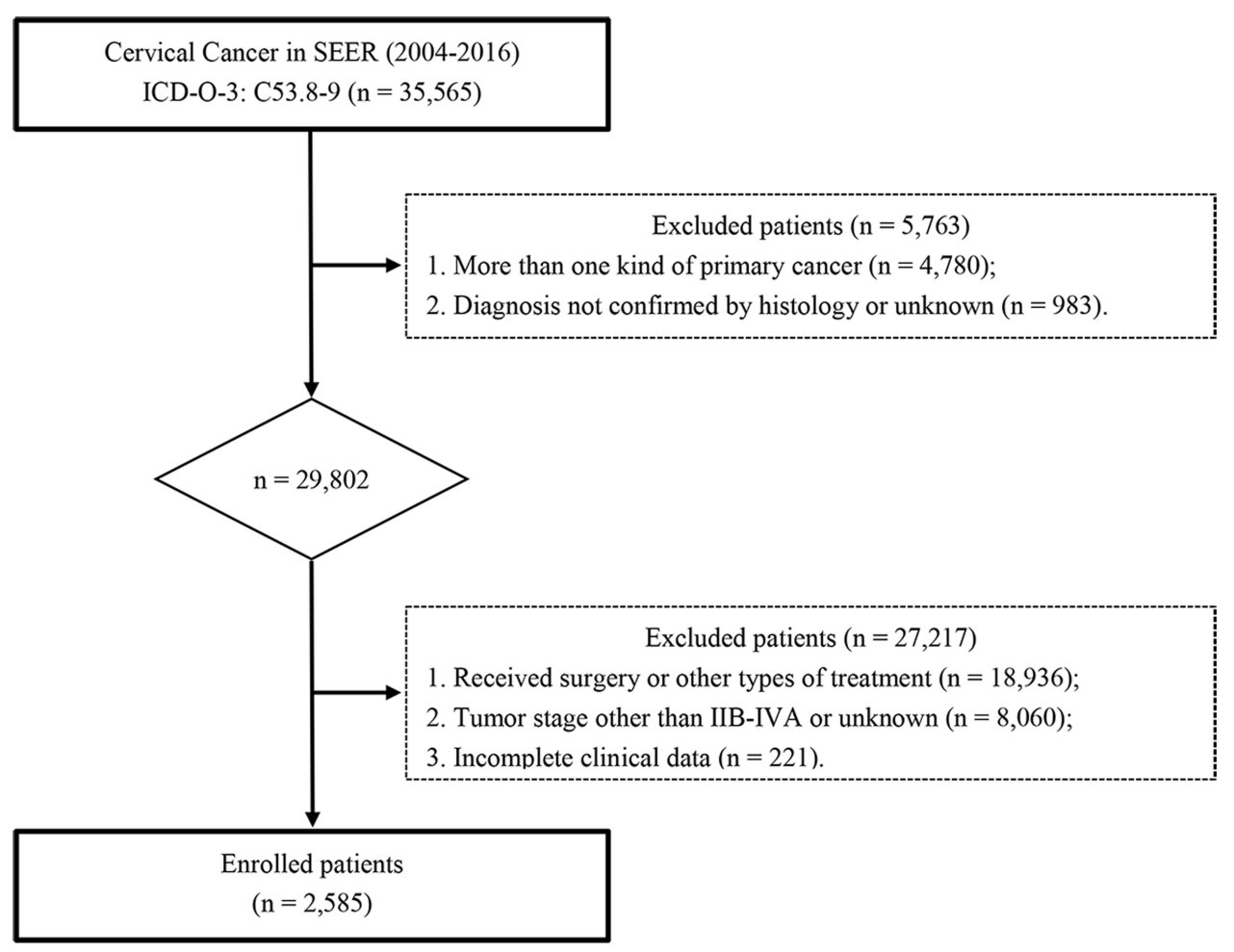

Figure I Patient selection flowchart.

characteristic (tdROC) with the nearest-neighbor estimation (NNE) method was employed to assess the prediction accuracy in evaluating 3- and 5-year OS rates and further compared with the DeLong's test with the survivalROC and $p R O C$ packages in the R platform. ${ }^{26,27}$ Differences were considered significant when the two-sided $P$-values were less than 0.05 .

Statistical analyses were conducted using the R software, version 3.6.2 (Institute for Statistics and Mathematics, Vienna, Austria; https://www.r-project.org) and IBM Statistical Package for the Social Sciences (SPSS) software, version 25.0 (IBM Corporation, Armonk, NY, USA).

\section{Results}

\section{Demographic and Baseline Characteristics}

Data of 2585 eligible patients with CC were extracted from the SEER database between 2004 and 2016. The demographic and baseline characteristics of the patients are summarized in Table 1. In the 2014 FIGO staging system, the numbers of patients with stage IIB, IIIA, IIIB and IVA disease were 1448 (56.0\%), 161 (6.2\%), 868 (33.6\%) and 108 (4.2\%), respectively. In the 2018 FIGO staging system, the number of patients with stage IIB, IIIA, IIIB and IVA disease were 1079 (41.7\%), 95 (3.7\%), $542(21.0 \%), 761$ (29.4\%) and 108 (4.2\%), respectively.

Among 761 patients with stage IIIC1 disease, 369 (48.5\%) with stage IIB disease in the 2014 FIGO staging system were upstaged to stage IIIC1, followed by $42.8 \%(\mathrm{n}=326)$ patients with stage IIIB disease and $8.7 \%$ patients $(\mathrm{n}=66)$ with stage IIIA disease (Figure 2A). Additionally, in the initial 2014 FIGO staging system (Figure 2B), patients with 2014 FIGO stage IIIA $(66 / 161,41.0 \%)$ and stage IIIB $(326 / 868,37.6 \%)$ disease constituted the major stage IIIC1 patient population, whereas patients with stage IIB disease in 2014 FIGO staging system constituted $25.5 \%(369 / 1448)$ of the total patient population despite the statistical significance between stage IIIA/IIIB and stage IIB in the Bonferroni's test $(P<0.001)$.

\section{Survival Outcomes}

The 3- and 5-year OS rates were 63.9\% (95\% confidence interval [CI], 0.619-0.659) and 56.6\% (95\% CI, 0.544-0.588), respectively, with a median OS of 98 months $(95 \% \mathrm{CI}$, not reached) for the entire cohort. 
Table I Baseline Characteristics of Stage IIB-IVA Cervix Carcinoma Patients

\begin{tabular}{|c|c|}
\hline Characteristic & $\begin{array}{c}\text { Frequency } \\
\text { (\%) }\end{array}$ \\
\hline \multicolumn{2}{|l|}{ Age at diagnosis (years) } \\
\hline Median (min-max) & 51 (19-97) \\
\hline$<70$ & $2276(88.0)$ \\
\hline$\geq 70$ & $309(12.0)$ \\
\hline \multicolumn{2}{|l|}{ Marital status } \\
\hline Married & $983(38.0)$ \\
\hline Unmarried and others & $1602(62.0)$ \\
\hline \multicolumn{2}{|l|}{ Race } \\
\hline White & $1865(72.1)$ \\
\hline Black & $381(14.7)$ \\
\hline Others & $339(13.2)$ \\
\hline \multicolumn{2}{|l|}{ Histology } \\
\hline SCC & $2243(86.8)$ \\
\hline$A C$ & $176(6.8)$ \\
\hline Others & $166(6.4)$ \\
\hline \multicolumn{2}{|l|}{ Differentiation } \\
\hline Well or moderately differentiated & $931(36.0)$ \\
\hline Poorly or undifferentiated & $934(36.1)$ \\
\hline Unknown & $720(27.9)$ \\
\hline \multicolumn{2}{|l|}{ Tumor size $(\mathrm{mm})$} \\
\hline$<60$ & $771(29.8)$ \\
\hline$\geq 60$ & $1015(39.3)$ \\
\hline Unknown & 799 (30.9) \\
\hline \multicolumn{2}{|l|}{2014 FIGO stage } \\
\hline IIB & I $448(56.0)$ \\
\hline IIIA & $|6|(6.2)$ \\
\hline IIIIB & $868(33.6)$ \\
\hline IVA & $108(4.2)$ \\
\hline \multicolumn{2}{|l|}{2018 FIGO stage } \\
\hline IIB & $1079(41.7)$ \\
\hline IIIA & $95(3.7)$ \\
\hline IIIIB & $542(21.0)$ \\
\hline $\mathrm{IIICl}$ & $761(29.4)$ \\
\hline IVA & $108(4.2)$ \\
\hline \multicolumn{2}{|l|}{ Chemotherapy (CT) } \\
\hline No/Unknown & $194(7.5)$ \\
\hline Yes & $2391(92.5)$ \\
\hline
\end{tabular}

Abbreviations: SCC, squamous cell carcinoma; AC, adenocarcinoma; FIGO, International Federation of Gynecology and Obstetrics.

In the 2014 FIGO staging system, the median OS of patients in stage IIB, IIIA, IIIB and IVA groups was as follows: not observed, 74 months (95\% CI, 31.6-116.4), 46 months (95\% CI, 35.2-56.8) and 25 months (95\% CI, 17.3-32.7), respectively, whereas the 3-year OS rates were as follows: $72.8 \%$ (95\% CI, 0.703-0.753), $61.0 \%$ (95\% CI, 0.527-0.692), $52.6 \%$ (95\% CI, 0.491-0.561) and 42.3\% (95\% CI, 0.315-0.531), respectively. The corresponding 5-year OS rates were as follows: $65.4 \%(95 \% \mathrm{CI}, 0.625-0.683), 50.5 \%$ (95\% CI, $0.417-0.592), 45.5 \%$ (95\% CI, $0.418-0.492)$ and $34.1 \%$ (95\% CI, 0.228-0.454), respectively. Figure 3A displays the Kaplan-Meier survival curve and Log rank tests of patients with stage IIB-IVA CC, which is stratified according to the 2014 FIGO staging system. Apart from the patients with 


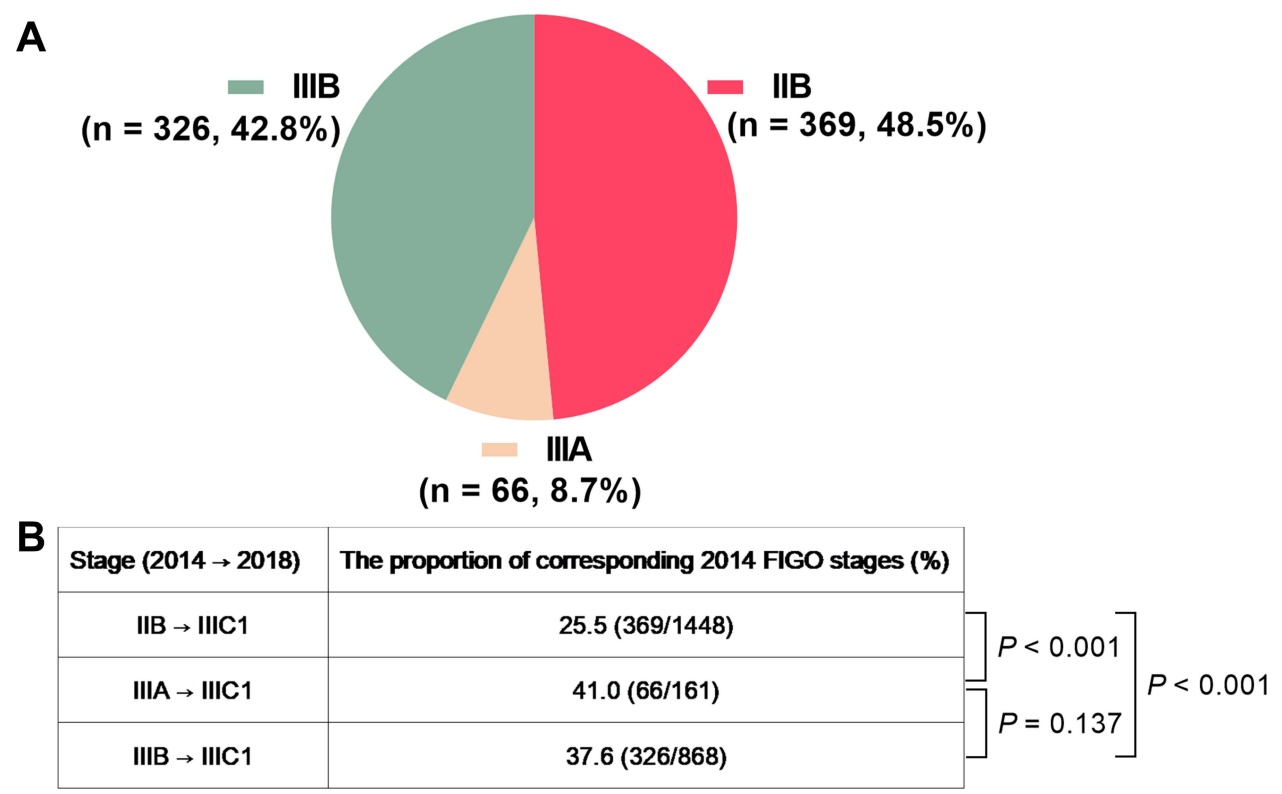

Figure 2 (A) The proportion of patients diagnosed with 2018 FIGO stage IIICI; (B) comparison among the patients with 2014 FIGO stage IIB-IIIB diseases who were upstaged to 2018 FIGO stage IIICl.
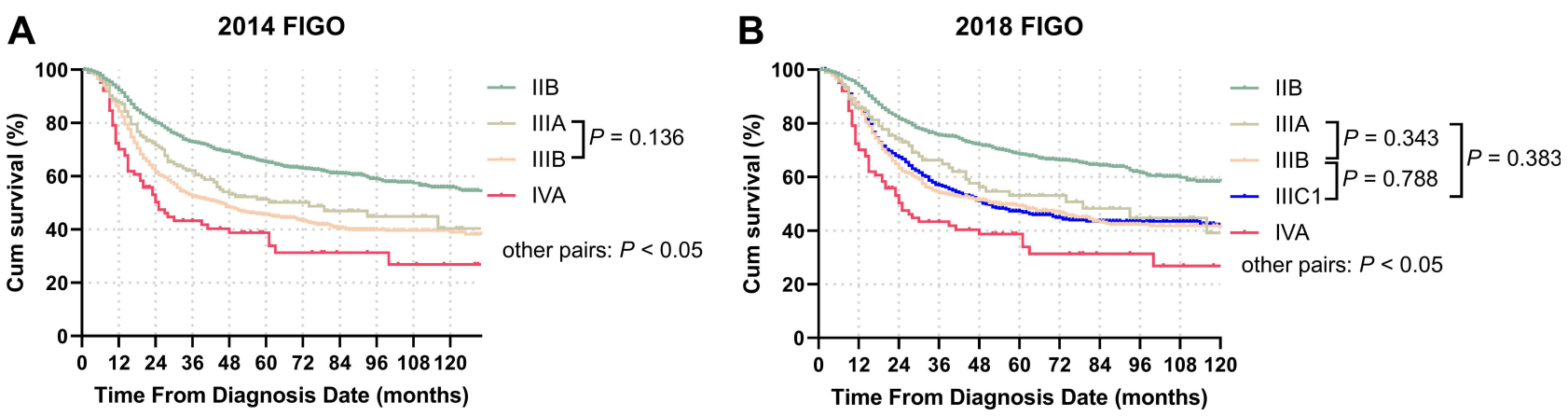

Figure 3 Overall survival (OS) of patients with stage IIB-IVA disease according to (A) the 2014 FIGO staging system and (B) the $2018 \mathrm{FIGO}$ staging system.

stage IIIA disease who revealed a non-significant survival benefit over patients with stage IIIB disease $(P=0.136)$, the other groups showed statistically significant differences $(P<0.05)$.

In the 2018 FIGO staging system, the median OS times of patients in stage IIB, IIIA, IIIB and IIIC1 groups were as follows: not observed, 79 months (95\% CI, 31.5-126.5), 55 months (95\% CI, 35.0-75.0) and 50 months (95\% CI, 37.362.7), respectively, and the 3-year OS rates were as follows: $75.5 \%$ (95\% CI, $0.728-0.782$ ), $65.1 \%$ (95\% CI, 0.549 $0.753), 54.2 \%$ ( $95 \% \mathrm{CI}, 0.497-0.587)$, and $56.9 \%$ (95\% CI, 0.530-0.608), respectively. The corresponding 5-year OS rates were as follows: $68.5 \%$ (95\% CI, $0.654-0.716), 52.7 \%$ (95\% CI, $0.415-0.639), 49.3 \%$ (95\% CI, $0.445-0.540)$, and $46.8 \%$ (95\% CI, 0.425-0.511), respectively. The survival outcomes of the patients in the IVA group were consistent with those of the 2014 FIGO staging system (Figure 3B). Moreover, a significant difference was not observed among stage III subgroups (IIIA versus IIIB: $P=0.343$; IIIA versus IIIC1: $P=0.383$ and IIIB versus IIIC1: $P=0.788$ ).

\section{Prognostic Factors for OS}

Univariate Cox regression analysis revealed that all clinicopathological and treatment-related characteristics were significantly associated with OS. In the multivariate analysis that correlated with the 2014 FIGO staging system, the significant variables were as follows: age at diagnosis ( $<70$ years versus $\geq 70$ years, $P<0.001$, hazard ratio [HR] $=1.466$, $95 \% \mathrm{CI}, 1.225-1.754$ ), tumor histology (squamous cell carcinoma $[\mathrm{SCC}]$ versus adenocarcinoma $[\mathrm{AC}], P=0.011, \mathrm{HR}=$ 
1.341, 95\% CI, 1.069-1.683; SCC versus others, $P<0.001$, HR $=1.628,95 \%$ CI, 1.296-2.046), tumor differentiation (well or moderately differentiated versus poorly or undifferentiated, $P=0.001$, HR $=1.285,95 \%$ CI, 1.110-1.487; well or moderately differentiated versus unknown, $P=0.822 \mathrm{HR}=1.019,95 \% \mathrm{CI}, 0.865-1.201)$, tumor size $(<60 \mathrm{~mm}$ versus $\geq 60 \mathrm{~mm}, P=0.033$, HR $=1.193,95 \% \mathrm{CI}, 1.015-1.402 ;<60 \mathrm{~mm}$ versus unknown, $P=0.009$, HR $=1.247,95 \% \mathrm{CI}$, 1.057-1.471), 2014 FIGO stage (IIB versus IIIA, $P=0.001$, HR $=1.509,95 \%$ CI, $1.171-1.945$; IIB versus IIIB, $P<$ $0.001, \mathrm{HR}=1.826,95 \% \mathrm{CI}, 1.597-2.087$; IIB versus IVA, $P<0.001, \mathrm{HR}=2.387,95 \% \mathrm{CI}, 1.803-3.161$ ) and CT (None/ unknown versus Yes, $P=0.019, \mathrm{HR}=0.767,95 \% \mathrm{CI}, 0.615-0.957)$. Similarly, the same variables were selected based on the multivariate Cox regression model that correlated with the 2018 FIGO staging system with a slight change in statistical differences (Table 2).

\section{tdROC Comparison of 3- and 5-Year OS Between the Two Staging Systems}

Subsequently, we compared the prediction accuracy of the 2014 FIGO and 2018 FIGO staging systems in evaluating 3and 5-year OS. In the earlier staging system (2014 version), the area under the curve (AUC) of tdROC for 3- and 5-year OS was 0.614 and 0.613, respectively, whereas, for the 2018 FIGO staging system, AUC values were 0.603 and 0.613 , respectively (Figure 4A and B). DeLong's tests revealed that there were no statistical differences in the two different staging systems for patients who underwent EBRT and BRT $(P=0.912$ for 3-year and $P=0.863$ for 5-year OS, respectively).

\section{Discussion}

The purpose of the present study was to explore the prognostic significance of parameters that are available in the SEER program on OS in patients with IIB-IVA CC based on the 2014 FIGO staging system who underwent EBRT and BRT. Factors including older age at diagnosis (>70 years), tumor histology other than SCC, tumor histology other than well or moderately differentiated, tumor size larger than $60 \mathrm{~mm}$ or unknown, advanced tumor stages and treatment without CT or unknown were significant parameters associated with decreased OS in patients who were registered to undergo EBRT and BRT. Moreover, when the tumor stage was converted into the 2018 FIGO staging system, the same factors with slightly different statistical values were observed in the multivariate analysis, which verified the stability of the Cox proportional hazards model. In a previous study, ${ }^{28}$ Tseng et al retrospectively reviewed the survival outcomes of 251 patients with stage IIB-IVA CC who were treated with CCRT. Multifactor Cox regression analysis revealed that advanced age at diagnosis, larger tumor size, higher serum SCC antigen levels and variables correlated with advanced tumor stages including positive parametrial invasion, lymph node metastasis, hydronephrosis and bladder/rectum invasion, were independent factors associated with a significantly worse 5-year OS. Another SEER analysis compared survival outcomes between SCC and AC in 4131 patients with CC. ${ }^{29}$ Tumor stages were recorded based on the 2009 FIGO staging system. In the multivariate Cox models, advanced tumor stage, combination treatment with EBRT and BRT and use of CT were proven to be significant factors associated with OS, which was partially consistent with the present study.

Furthermore, the prognostic accuracy of the 2014 and 2018 FIGO staging systems are compared in this study. Although various studies have proposed that prognostic prediction based on tumor stage alone is insufficient ${ }^{30-32}$ for patients with $\mathrm{CC}$ who underwent nonsurgical treatment, a single variable with a prediction accuracy of 3- and 5-year OS exceeding 0.6 suffices to exhibit its significant value in prediction analysis and requires further investigation. A similar application using the SEER database was reported by Matsuo et al. In the subgroup comparison, 11,733 patients with CC diagnosed with stage III disease were enrolled. Among them, 6888 patients were restaged with stage IIIC1 based on the 2018 FIGO staging system; however, stage IIIC2 data were untraced in the database. ${ }^{33}$ In the multivariable analysis with cause-specific survival (CSS), stage IIIC1 was demonstrated to be independently associated with significantly improved CSS compared to stage IIIA ( $P=0.018, \mathrm{HR}=0.88,95 \% \mathrm{CI}, 0.70-0.98$, ) and stage IIIB $(P<0.001, \mathrm{HR}=0.79,95 \% \mathrm{CI}$, 0.74-0.85), respectively. Subsequently, the authors compared the survival outcomes solely based on T-stage, despite the LN metastatic status in patients with stage IIIC1 disease. The Kaplan-Meier curve revealed that the 5-year CSS rates were $74.8 \%$ for $\mathrm{T} 1,58.7 \%$ for $\mathrm{T} 2$ and $39.3 \%$ for T3, indicating significant differences among various groups $(P<0.001)$. T-stage remained an independent prognostic parameter for CSS $(P<0.001)$ on multivariable Cox analysis. In the aforementioned Korean study, ${ }^{18}$ the authors further explored the distribution of the 2014 FIGO stages in the 2018 FIGO 
Table 2 Univariate and Multivariate Analysis of OS in Cervix Carcinoma Patients by Using Cox Regression Model

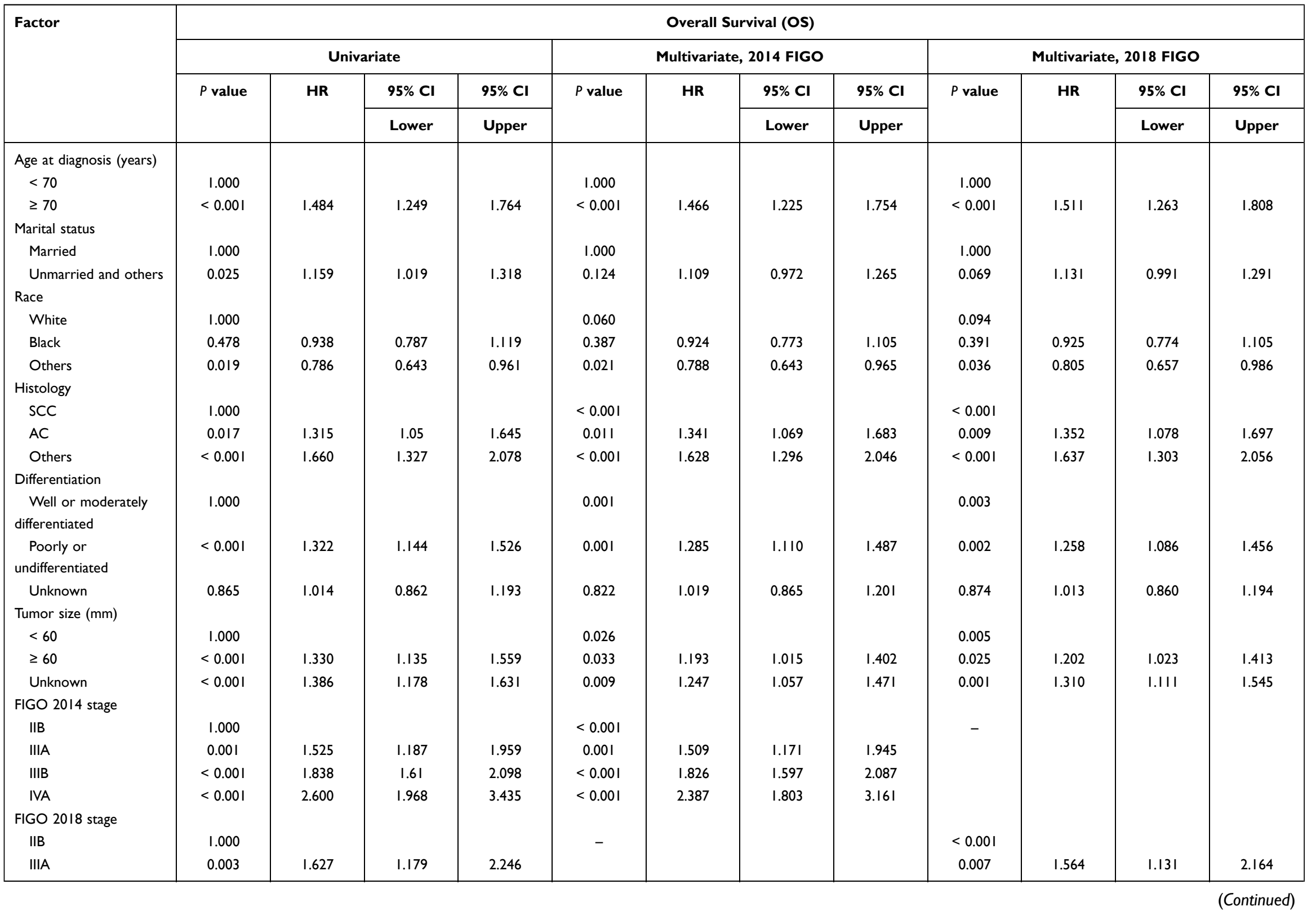


Table 2 (Continued)

\begin{tabular}{|c|c|c|c|c|c|c|c|c|c|c|c|c|}
\hline \multirow[t]{4}{*}{ Factor } & \multicolumn{12}{|c|}{ Overall Survival (OS) } \\
\hline & \multicolumn{4}{|c|}{ Univariate } & \multicolumn{4}{|c|}{ Multivariate, 2014 FIGO } & \multicolumn{4}{|c|}{ Multivariate, 2018 FIGO } \\
\hline & \multirow[t]{2}{*}{$P$ value } & \multirow[t]{2}{*}{ HR } & \multirow{2}{*}{$\begin{array}{l}95 \% \mathrm{Cl} \\
\text { Lower }\end{array}$} & \multirow{2}{*}{$\begin{array}{l}95 \% \mathrm{Cl} \\
\text { Upper }\end{array}$} & \multirow[t]{2}{*}{$P$ value } & \multirow[t]{2}{*}{ HR } & \multirow{2}{*}{$\begin{array}{l}95 \% \mathrm{Cl} \\
\text { Lower }\end{array}$} & \multirow{2}{*}{$\begin{array}{l}95 \% \mathrm{Cl} \\
\text { Upper }\end{array}$} & \multirow[t]{2}{*}{$P$ value } & \multirow[t]{2}{*}{ HR } & $95 \% \mathrm{Cl}$ & $95 \% \mathrm{Cl}$ \\
\hline & & & & & & & & & & & Lower & Upper \\
\hline IIIB & $<0.001$ & 1.905 & 1.617 & 2.243 & & & & & $<0.001$ & 1.862 & 1.579 & 2.195 \\
\hline $\mathrm{IIICl}$ & $<0.001$ & 1.878 & 1.608 & 2.193 & & & & & $<0.001$ & 1.942 & 1.659 & 2.273 \\
\hline IVA & $<0.001$ & 2.897 & 2.179 & 3.852 & & & & & $<0.001$ & 2.670 & 2.003 & 3.558 \\
\hline \multicolumn{13}{|l|}{ Chemotherapy } \\
\hline None/unknown & 1.000 & & & & 1.000 & & & & 1.000 & & & \\
\hline Yes & 0.003 & 0.721 & 0.582 & 0.895 & 0.019 & 0.767 & 0.615 & 0.957 & 0.022 & 0.774 & 0.621 & 0.964 \\
\hline
\end{tabular}

Abbreviations: $\mathrm{HR}$, hazard ratio; $\mathrm{Cl}$, confidence interval. 

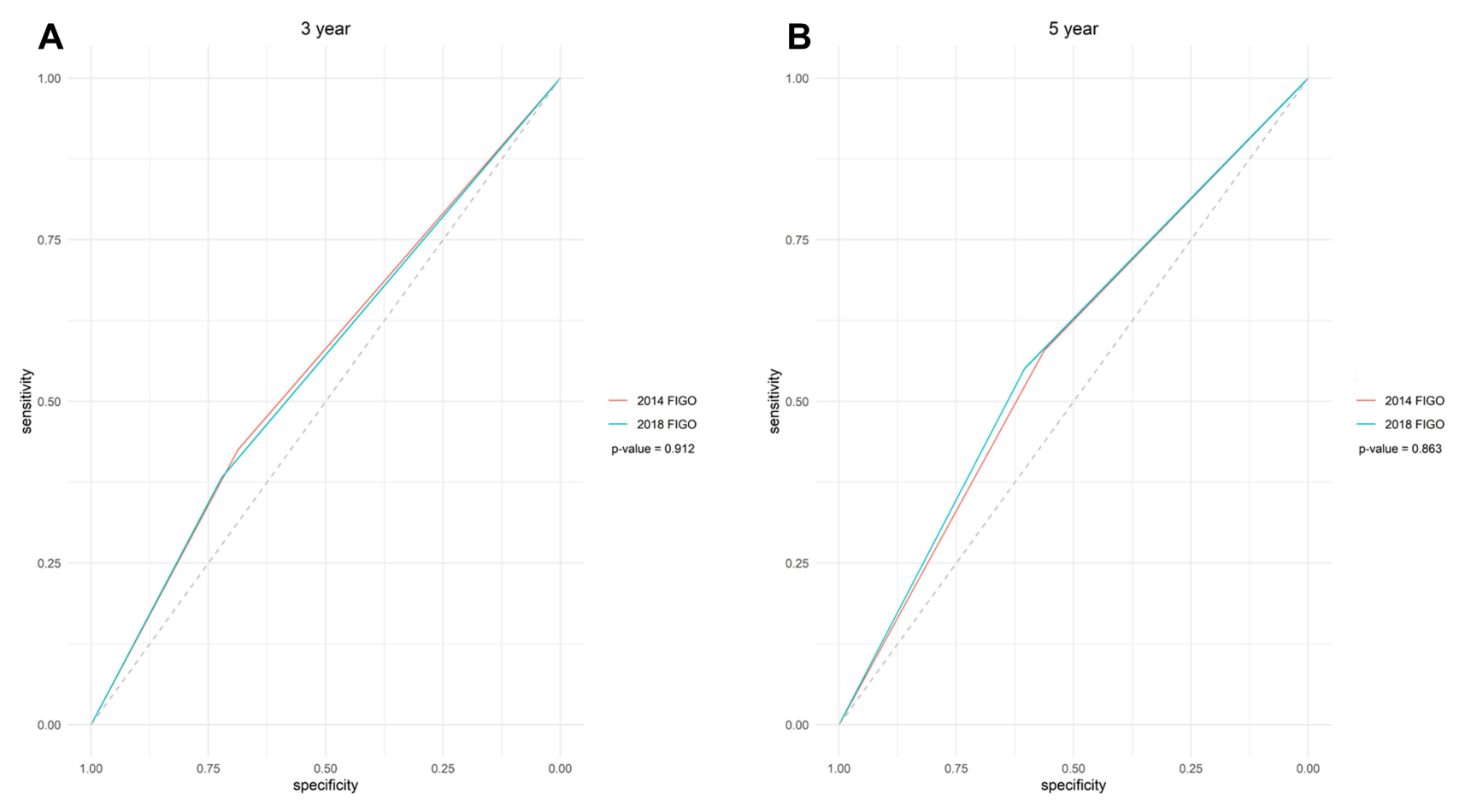

Figure 4 tdROC curves of the 2014 and $2018 \mathrm{FIGO}$ staging systems at (A) 3-year OS and (B) 5-year OS.

stage IIIC. Great heterogeneity with a wide range of stages of the 2014 FIGO staging system was documented. In both stage IIIC1 and stage IIIC2, stage IIB in the 2014 FIGO staging system constituted $41 \%$ and $54 \%$ of patients with stage IIIC1 and stage IIIC2 disease, respectively. Stage IB1 (17\%) ranked second place in stage IIIC1, whereas stage IIIB (28\%) ranked second place in stage IIIC2 based on the definition of the 2018 FIGO staging system. In the present study, based on the 2014 FIGO staging system, patients with stage IIIA (41.0\%) and IIIB (37.6\%) disease constituted a major proportion of the patient population, whereas patients with stage IIB disease constituted $25.5 \%$ of the patient population, with significant difference between stages IIIA/IIIB and IIB diseases. Moreover, survival analysis of the 2018 FIGO staging system further revealed that there was no statistical significance among the stage III subgroups. Furthermore, DeLong's test revealed no significant differences between the nearly overlapping prediction accuracy of the two systems in evaluating 3- and 5-year OS based on tdROC curves. This lack of significant difference could be attributed to the following three reasons: as shown in Figure 2B, patients with stage IIIA/IIIB disease have a larger proportion accompanied by LN metastasis compared to those with stage IIB disease, which was substantiated by other largesample retrospective analysis. ${ }^{34-36}$ Therefore, distinguishing patients with stage IIIA/IIIB disease into single-stage IIIC1 was difficult. Moreover, for patients with stage IIB-IVA disease who underwent EBRT and BRT, whole pelvic irradiation was the recommended treatment strategy by both FIGO and NCCN despite the status of pelvic LNs. Previous studies have revealed that in patients with positive LNs metastasis, a concurrent boost for the LNs during EBRT can achieve satisfactory local tumor controls with acceptable toxicities. ${ }^{37-39}$ Lastly, stage IIIA constituted the smallest proportion of patients in both the 2014 and 2018 FIGO staging systems, which might also cause a bias in survival comparisons. Therefore, classifying patients with indications of LN metastasis into a single-stage IIIC could result in significant heterogeneity with variable survival outcomes and require further verification in clinic settings. ${ }^{16,17}$

Owing to the status of para-aortic LN metastasis could not be traced in the SEER program and lack of data on stage IIIC2 disease was the major limitation in the present study. Other important variables for patients who underwent EBRT and BRT, such as baseline characteristics (haemoglobin level; performance status; staging methods by clinical or surgical approaches; the status of HPV infection) and treatment-related factors (RT modality, CT sequence and dosage), which were unavailable in the SEER database. Lastly, the Cox regression model is the most frequently reported survival prediction method used not only in the present study but also in the majority of studies mentioned in the discussion. 
Previously, Matsuo et al evaluated advanced statistical methods using a deep-learning model, which was based on the data analysis of 768 patients with CC, with each patient having 40 features. The results suggested better survival prediction with the deep-learning neural network model. ${ }^{40}$ However, in the present study, each patient had only eight features, which precluded us from using advanced analytical models; however, such advanced models can be evaluated in future studies.

In conclusion, we assessed 2585 previously diagnosed patients with stage IIB-IVA CC based on the 2014 FIGO staging system who underwent EBRT and BRT and compared the prediction accuracy of the 2014 FIGO and 2018 FIGO staging systems in evaluating 3- and 5-year OS. Multivariate Cox analysis confirmed that both staging systems were robust prognostic factors for OS. However, owing to substantial heterogeneity between different tumor stages, a wide range of survival outcomes were observed in the present study, and the impact of pelvic LN metastasis on OS was not observed, and factors such as tumor sizes, radiomics features and dynamic tumor shrinkages should be further evaluated using a large sample size.

\section{Data Sharing Statement}

The datasets generated for this study are available on request to the corresponding author.

\section{Ethics Statement}

Given that data in the SEER database have been de-identified and are publicly available after permission, written informed consent for participation was not required for this study. The Institutional Review Board of Zhejiang Provincial People's Hospital exempted this study from ethical review. We confirm that this study was performed in accordance with the Declaration of Helsinki.

\section{Acknowledgments}

We thank the National Cancer Institute (NCI) for providing the SEER data after permission. We thank the Bullet Edits Ltd. for their help with English editing.

\section{Author Contributions}

All authors made substantial contributions to conception and design, acquisition of data, or analysis and interpretation of data; took part in drafting the article or revising it critically for important intellectual content; agreed to submit to the current journal; gave final approval for the version to be published; and agreed to be accountable for all aspects of the work.

\section{Funding}

There was no funding support for this study.

\section{Disclosure}

The authors declare that they have no conflicts of interest for this work.

\section{References}

1. Sung H, Ferlay J, Siegel RL, et al. Global Cancer Statistics 2020: GLOBOCAN estimates of incidence and mortality worldwide for 36 cancers in 185 countries. CA Cancer J Clin. 2021;71(3):209-249. doi:10.3322/caac.21660

2. Bray F, Ferlay J, Soerjomataram I, Siegel RL, Torre LA, Jemal A. Global cancer statistics 2018: GLOBOCAN estimates of incidence and mortality worldwide for 36 cancers in 185 countries. CA Cancer J Clin. 2018;68(6):394-424. doi:10.3322/caac.21492

3. Ferrandina G, Gallotta V, Federico A, et al. Minimally invasive approaches in locally advanced cervical cancer patients undergoing radical surgery after chemoradiotherapy: a propensity score analysis. Ann Surg Oncol. 2021;28(7):3616-3626. doi:10.1245/s10434-020-09302-y

4. Lin S, Gao K, Gu S, et al. Worldwide trends in cervical cancer incidence and mortality, with predictions for the next 15 years. Cancer. 2021;127 (21):4030-4039.

5. Morris M, Eifel PJ, Lu J, et al. Pelvic radiation with concurrent chemotherapy compared with pelvic and para-aortic radiation for high-risk cervical cancer. N Engl J Med. 1999;340(15):1137-1143. doi:10.1056/NEJM199904153401501

6. Whitney CW, Sause W, Bundy BN, et al. Randomized comparison of fluorouracil plus cisplatin versus hydroxyurea as an adjunct to radiation therapy in stage IIB-IVA carcinoma of the cervix with negative para-aortic lymph nodes: a Gynecologic Oncology Group and Southwest Oncology Group study. J Clin Oncol. 1999;17(5):1339-1348. doi:10.1200/JCO.1999.17.5.1339 
7. Eifel PJ, Winter K, Morris M, et al. Pelvic irradiation with concurrent chemotherapy versus pelvic and para-aortic irradiation for high-risk cervical cancer: an update of radiation therapy oncology group trial (RTOG) 90-01. J Clin Oncol. 2004;22(5):872-880. doi:10.1200/JCO.2004.07.197

8. Peiretti M, Zapardiel I, Zanagnolo V, Landoni F, Morrow CP, Maggioni A. Management of recurrent cervical cancer: a review of the literature. Surg Oncol. 2012;21(2):e59-66. doi:10.1016/j.suronc.2011.12.008

9. Boussios S, Seraj E, Zarkavelis G, et al. Management of patients with recurrent/advanced cervical cancer beyond First line platinum regimens: where do we stand? A literature review. Crit Rev Oncol Hematol. 2016;108:164-174. doi:10.1016/j.critrevonc.2016.11.006

10. Antoch G, Vogt FM, Freudenberg LS, et al. Whole-body dual-modality PET/CT and whole-body MRI for tumor staging in oncology. JAMA. 2003;290(24):3199-3206. doi:10.1001/jama.290.24.3199

11. Oncology FCoG. FIGO staging for carcinoma of the vulva, cervix, and corpus uteri. Int J Gynaecol Obstet. 2014;125(2):97-98. doi:10.1016/j. ijgo.2014.02.003

12. Bhatla N, Berek JS, Cuello Fredes M, et al. Revised FIGO staging for carcinoma of the cervix uteri. Int J Gynaecol Obstet. 2019;145(1):129-135. doi:10.1002/ijgo.12749

13. Saleh M, Virarkar M, Javadi S, Elsherif SB, de Castro Faria S, Bhosale P. Cervical cancer: 2018 revised international federation of gynecology and obstetrics staging system and the role of imaging. AJR Am J Roentgenol. 2020;214(5):1182-1195. doi:10.2214/AJR.19.21819

14. Salib MY, Russell JHB, Stewart VR, et al. 2018 FIGO staging classification for cervical cancer: added benefits of imaging. Radiographics. 2020;40 (6): 1807-1822. doi:10.1148/rg.2020200013

15. Liu X, Wang J, Hu K, et al. Validation of the 2018 FIGO staging system of cervical cancer for stage III patients with a cohort from China. Cancer Manag Res. 2020;12:1405-1410. doi:10.2147/CMAR.S239624

16. Salvo G, Odetto D, Pareja R, Frumovitz M, Ramirez PT. Revised 2018 International Federation of Gynecology and Obstetrics (FIGO) cervical cancer staging: a review of gaps and questions that remain. Int J Gynecol Cancer. 2020;30(6):873-878. doi:10.1136/ijgc-2020-001257

17. Olthof EP, Mom CH, van der Velden J. More attention is needed for the corrigendum to the revised FIGO staging for carcinoma of the cervix uteri. Int J Gynecol Cancer. 2020;30(11):1850. doi:10.1136/ijgc-2020-001959

18. Shin W, Ham TY, Park YR, Lim MC, Won YJ. Comparing survival outcomes for cervical cancer based on the 2014 and 2018 International Federation of Gynecology and Obstetrics staging systems. Sci Rep. 2021;11(1):6988. doi:10.1038/s41598-021-86283-2

19. Sharma C, Deutsch I, Horowitz DP, et al. Patterns of care and treatment outcomes for elderly women with cervical cancer. Cancer. 2012;118 (14):3618-3626. doi:10.1002/cncr.26589

20. Nogueira-Rodrigues A, de Melo AC, Garces AH, et al. Patterns of care and outcome of elderly women diagnosed with cervical cancer in the developing world. Int J Gynecol Cancer. 2016;26(7):1246-1251. doi:10.1097/IGC.0000000000000756

21. Kobayashi D, Okonogi N, Wakatsuki M, et al. Impact of CT-based brachytherapy in elderly patients with cervical cancer. Brachytherapy. 2019;18 (6):771-779. doi:10.1016/j.brachy.2019.08.002

22. Yang D, Hanna DL, Usher J, et al. Impact of sex on the survival of patients with hepatocellular carcinoma: a surveillance, epidemiology, and end results analysis. Cancer. 2014;120(23):3707-3716. doi:10.1002/cncr.28912

23. Ellis L, Canchola AJ, Spiegel D, Ladabaum U, Haile R, Gomez SL. Racial and ethnic disparities in cancer survival: the contribution of tumor, sociodemographic, institutional, and neighborhood characteristics. J Clin Oncol. 2018;36(1):25-33. doi:10.1200/JCO.2017.74.2049

24. Frederick L, Page DL, Fleming ID, et al. American Joint Committee on Cancer (AJCC) Cancer Staging Manual. 6th ed. New York, NY: Springer; 2002.

25. Song T, Wan Q, Fang M, Zhan W, Xu H, Shou H. Trends and predictors of survival for small cell carcinoma of the cervix uteri: a SEER population study. Eur J Obstet Gynecol Reprod Biol. 2020;253:35-41. doi:10.1016/j.ejogrb.2020.07.054

26. DeLong ER, DeLong DM, Clarke-Pearson DL. Comparing the areas under two or more correlated receiver operating characteristic curves: a nonparametric approach. Biometrics. 1988;44(3):837-845. doi:10.2307/2531595

27. Robin X, Turck N, Hainard A, et al. pROC: an open-source package for R and $\mathrm{S}+$ to analyze and compare ROC curves. BMC Bioinform. 2011;12:77. doi:10.1186/1471-2105-12-77

28. Tseng JY, Yen MS, Twu NF, et al. Prognostic nomogram for overall survival in stage IIB-IVA cervical cancer patients treated with concurrent chemoradiotherapy. Am J Obstet Gynecol. 2010;202(2):174 e171-177. doi:10.1016/j.ajog.2009.09.028

29. Tian T, Gong X, Gao X, Li Y, Ju W, Ai Y. Comparison of survival outcomes of locally advanced cervical cancer by histopathological types in the surveillance, epidemiology, and end results (SEER) database: a propensity score matching study. Infect Agent Cancer. 2020;15:33. doi:10.1186/ s13027-020-00299-3

30. Lucia F, Visvikis D, Desseroit MC, et al. Prediction of outcome using pretreatment (18) F-FDGPET/CT and MRI radiomics in locally advanced cervical cancer treated with chemoradiotherapy. Eur J Nucl Med Mol Imaging. 2018;45(5):768-786. doi:10.1007/s00259-017-3898-7

31. Raut A, Chopra S, Mittal P, et al. FIGO classification 2018: validation study in patients with locally advanced cervix cancer treated with chemoradiation. Int J Radiat Oncol Biol Phys. 2020;108(5):1248-1256. doi:10.1016/j.ijrobp.2020.07.020

32. Zhang J, Rashmi R, Inkman M, et al. Integrating imaging and RNA-seq improves outcome prediction in cervical cancer. J Clin Invest. 2021;131(5). doi:10.1172/JCI139232

33. Matsuo K, Machida H, Mandelbaum RS, Konishi I, Mikami M. Validation of the 2018 FIGO cervical cancer staging system. Gynecol Oncol. 2019;152(1):87-93. doi:10.1016/j.ygyno.2018.10.026

34. Kidd EA, Siegel BA, Dehdashti F, et al. Lymph node staging by positron emission tomography in cervical cancer: relationship to prognosis. $J$ Clin Oncol. 2010;28(12):2108-2113. doi:10.1200/JCO.2009.25.4151

35. Graves S, Seagle BL, Strohl AE, Shahabi S, Nieves-Neira W. Survival after pelvic exenteration for cervical cancer: a National cancer database study. Int J Gynecol Cancer. 2017;27(2):390-395. doi:10.1097/IGC.0000000000000884

36. McComas KN, Torgeson AM, Ager BJ, et al. The variable impact of positive lymph nodes in cervical cancer: implications of the new FIGO staging system. Gynecol Oncol. 2020;156(1):85-92. doi:10.1016/j.ygyno.2019.10.025

37. Rash DL, Lee YC, Kashefi A, et al. Clinical response of pelvic and para-aortic lymphadenopathy to a radiation boost in the definitive management of locally advanced cervical cancer. Int J Radiat Oncol Biol Phys. 2013;87(2):317-322. doi:10.1016/j.ijrobp.2013.06.2031

38. Vargo JA, Kim H, Choi S, et al. Extended field intensity modulated radiation therapy with concomitant boost for lymph node-positive cervical cancer: analysis of regional control and recurrence patterns in the positron emission tomography/computed tomography era. Int J Radiat Oncol Biol Phys. 2014;90(5):1091-1098. doi:10.1016/j.jirobp.2014.08.013 
39. Dang YZ, Li P, Li JP, et al. Efficacy and toxicity of IMRT-based simultaneous integrated boost for the definitive management of positive lymph nodes in patients with cervical cancer. $J$ Cancer. 2019;10(5):1103-1109. doi:10.7150/jca.29301

40. Matsuo K, Purushotham S, Jiang B, et al. Survival outcome prediction in cervical cancer: cox models vs deep-learning model. Am J Obstet Gynecol. 2019;220(4):381 e381-381 e314. doi:10.1016/j.ajog.2018.12.030

\section{Publish your work in this journal}

The International Journal of Women's Health is an international, peer-reviewed open-access journal publishing original research, reports, editorials, reviews and commentaries on all aspects of women's healthcare including gynecology, obstetrics, and breast cancer. The manuscript management system is completely online and includes a very quick and fair peer-review system, which is all easy to use. Visit http://www. dovepress.com/testimonials.php to read real quotes from published authors.

Submit your manuscript here: https://www.dovepress.com/international-journal-of-womens-health-journal 